\title{
REMARKS ON THE CONVERGENCE OF NEWTON'S METHOD UNDER HÖLDER CONTINUITY CONDITIONS
}

\author{
IOANNIS K. ARGYROS
}

\begin{abstract}
We use a Newton-like iteration to solve the nonlinear operator equation in a Banach space. The basic assumption is that the Fréchet-derivative of the nonlinear operator is Hölder continuous on some open ball centered at the initial guess. Under natural assumptions, we prove linear convergence of the iteration to a locally unique solution of the nonlinear equation.
\end{abstract}

Introduction. We introduce the Newton-like iteration

$$
x_{n+1}=x_{n}-F^{\prime}\left(y_{n}\right)^{-1} F\left(x_{n}\right), \quad n=0,1,2, \ldots
$$

to solve the equation

$$
F(x)=0
$$

where $F$ is a nonlinear operator on a Banach space $X$. Here we assume that the Fréchet derivative $F^{\prime}(x)$ of $F$ is Hölder continuous on some ball

$$
U\left(x_{0}, r_{0}\right)=\left\{x \in X \mid\left\|x-x_{0}\right\|<r_{0}\right\}, \quad r_{0}>0 .
$$

The point $x_{0} \in x$ and the arbitrary points $y_{n}, n=0,1,2, \ldots$ are chosen sufficiently close to the desired solution. Then under natural assumptions we .

Received May 19, 1989.

(1980) A.M.S. classification codes: 65J15, 65H10.

Krey words and phrases: Newton-like iteration, Banach space, Fréchet-derivative. 
show that (1) converges linearly to a unique solution $x^{*}$ of (2) in $U\left(x_{0}, r\right)$ for some $r>0$.

Note that for $y_{n}=x_{n}$ we obtain Newton's iteration, whereas for $y_{n}=x_{0}$ we obtain the modified one. The computer will determine the $y_{n}$ 's as to minimize the effort each time.

Here is an incomplete list of the usual assumptions for the convergence of (1) to a solution of (2):

(a) the existence and boundedness of the second Fréchet derivative of $F$ (see, ex. [2], [3] and [4]).

(b) The assumption of analyticity which eliminates explicit mention of the second derivative [7].

(c) The case when the first Fréchet derivative of $F$ satisfies a Lipschitz condition. (See, ex. [5], [6] and the references there.)

Finally,

(d) various other assumptions mainly based on the possibility of replacing $F^{\prime}\left(x_{n}\right)^{-1}$ with a sequence of linear operators which are "close" in some sense to $F^{\prime}\left(x_{n}\right)^{-1}, n=0,1,2, \ldots$ (See, ex. [5], [6] and the references there.)

We will need the following definition.

Definition. We assume that $F$ is once Fréchet-differentiable [2] and $F^{\prime}(x)$ is the first Fréchet-derivative at a point $x \in X$. It is well known that $F^{\prime}(x) \in L(x)$, the space of bounded linear operators from $X$ to $X$. We say that the Fréchetderivative $F^{\prime}(x)$ is Hölder continuous over a domain $D \subset X$ if for some $c>0$, $p \in[0,1]$, and all $x, y \in D$,

$$
\left\|F^{\prime}(x)-F^{\prime}(y)\right\| \leq c\|x-y\|^{P},
$$

From now on we will find it more convenient to assume that $D=U\left(x_{0}, r_{0}\right)$ for some fixed $x_{0} \in x$ and $r_{0}>0$.

Lemma. Let $F^{\prime}(x)$ be Hölder continuous on $U\left(x_{0}, r_{0}\right)$ for some $x_{0}, r_{0}, p$ such that $x_{0} \in x, r_{0}>0$ and $p \in[0,1]$. Suppose that $F^{\prime}\left(x_{0}\right)$ has a bounded inverse. For any $b_{0}>\left\|F^{\prime}\left(x_{0}\right)^{-1}\right\|$, there is a number $r_{3} \leq \min \left\{1, r_{0}\right\}$ such that: 
(a) If $x \in U\left(x_{0}, r_{3}\right)$, then the linear operator $F^{\prime}(x)$ has bounded inverse and

$$
\left\|F^{\prime}(x)^{-1}\right\|<b_{0}
$$

(b) if $x_{i} \in U\left(x_{0}, r_{3}\right), i=1,2,3$ then

$$
\left\|F\left(x_{1}\right)-F\left(x_{2}\right)-F^{\prime}\left(x_{3}\right)\left(x_{1}-x_{2}\right)\right\| \leq \frac{1}{2 b_{0}}\left\|x_{1}-x_{2}\right\| .
$$

Proof. (a) If $x \in U\left(x_{0}, r_{0}\right)$, then

$$
\left\|F^{\prime}(x)-F^{\prime}\left(x_{0}\right)\right\| \leq c\left\|x-x_{0}\right\|^{p} .
$$

Choose $r_{1}>0$ such that

$$
0<r_{1} \leq \min \left(r_{0},\left(\frac{1}{4 b_{0} c}\right)^{1 / p}\right)
$$

then if $x \in U\left(x_{0}, r_{1}\right)$

$$
\left\|F^{\prime}(x)-F^{\prime}\left(x_{0}\right)\right\| \leq c\left\|x-x_{0}\right\|^{p} \leq c r_{1}^{p} \leq \frac{1}{4 b_{0}} .
$$

Since,

$$
\left\|F^{\prime}\left(x_{0}\right)^{-1}\right\| \cdot\left\|F^{\prime}(x)-F^{\prime}\left(x_{0}\right)\right\|<b_{0} \cdot \frac{1}{4 b_{0}}<1,
$$

by the Banach lemma $F^{\prime}(x)$ has a bounded inverse for $x \in U\left(x_{0}, r_{1}\right)$. Therefore, there exists an $r_{2}>0$, with

$$
0<r_{2} \leq r_{1}
$$

such that if $x \in U\left(x_{0}, r_{2}\right)$, then $\left\|F^{\prime}(x)^{-1}\right\|<b_{0}$.

That proves (4).

(b) If $r_{3}=\min \left\{1, r_{2}\right\}$ and $x_{i} \in U\left(x_{0}, r_{3}\right), i=1,2,3$, we first have

$$
\begin{aligned}
& F\left(x_{1}\right)-F\left(x_{2}\right)-F^{\prime}\left(x_{3}\right)\left(x_{1}-x_{2}\right) \\
= & \left(F\left(x_{1}\right)-F\left(x_{2}\right)-F^{\prime}\left(x_{0}\right)\left(x_{1}-x_{2}\right)\right)+\left(F^{\prime}\left(x_{0}\right)-F^{\prime}\left(x_{3}\right)\right)\left(x_{1}-x_{2}\right) \\
= & \int_{0}^{1}\left(F^{\prime}\left[t x_{1}+(1-t) x_{2}\right]-F^{\prime}\left(x_{0}\right)\right)\left(x_{1}-x_{2}\right) d t+\left(F^{\prime}\left(x_{0}\right)-F^{\prime}\left(x_{3}\right)\right)\left(x_{1}-x_{2}\right) .
\end{aligned}
$$


But,

$$
\begin{aligned}
\left\|\int_{0}^{1}\left(F^{\prime}\left[t x_{1}+(1-t) x_{2}\right]-F^{\prime}\left(x_{0}\right)\right)\left(x_{1}-x_{2}\right) d t\right\| & \leq c r_{3}^{p}\left\|x_{1}-x_{2}\right\| \\
& \leq \frac{1}{4 b_{0}}\left\|x_{1}-x_{2}\right\|
\end{aligned}
$$

and

$$
\begin{aligned}
\left\|\left(F^{\prime}\left(x_{0}\right)-F^{\prime}\left(x_{3}\right)\right)\left(x_{1}-x_{2}\right)\right\| & \leq c r_{3}^{p}\left\|x_{1}-x_{2}\right\| \\
& \leq \frac{1}{4 b_{0}}\left\|x_{1}-x_{2}\right\|
\end{aligned}
$$

Therefore,

$$
\left\|F\left(x_{1}\right)-F\left(x_{2}\right)-F^{\prime}\left(x_{3}\right)\left(x_{1}-x_{2}\right)\right\| \leq \frac{1}{2 b_{0}}\left\|x_{1}-x_{2}\right\| .
$$

That proves (5) and completes the proof of the lemma.

We now state and prove the main result.

Theorem. Let $F^{\prime}(x)$ be Hölder continuous on $U\left(x_{0}, r_{3}\right)$, where $r_{3}$ is defined in the lemma. Suppose that $x_{0} \in X$ is such that $F^{\prime}\left(x_{0}\right)$ has an inverse satisfying $\left\|F^{\prime}\left(x_{0}\right)^{-1}\right\|<b_{0}<\infty$ and

$$
\left\|F\left(x_{0}\right)\right\|<\frac{r_{3}}{2 b_{0}} .
$$

Let $y_{n}$ be arbitrary points such that $y_{n} \in U\left(x_{0}, r_{3}\right), n=0,1,2, \ldots$

Then the iteration $\left[x_{n}\right]$, given by

$$
x_{n+1}=x_{n}-\left(F^{\prime}\left(y_{n}\right)\right)^{-1} F\left(x_{n}\right), \quad n=0,1,2, \ldots
$$

converges to a unique solution $x^{*}$ of (2) in $U\left(x_{0}, r_{3}\right)$.

Moreover, the following estimate holds

$$
\left\|x_{n}-x^{*}\right\|<2^{-n} r_{3}, \quad n=0,1,2, \ldots
$$

Proof. Using (1) for $y_{0}=x_{0}$ we obtain

$\left\|x_{1}-x_{0}\right\|=\left\|F^{\prime}\left(x_{0}\right)^{-1} F\left(x_{0}\right)\right\| \leq\left\|F^{\prime}\left(x_{0}\right)^{-1}\right\| \cdot\left\|F\left(x_{0}\right)\right\| \leq b_{0}\left\|F\left(x_{0}\right)\right\|<\frac{r_{3}}{2}$. 
By (5) and the identity

$$
F\left(x_{1}\right)=F\left(x_{1}\right)-F\left(x_{0}\right)-F^{\prime}\left(y_{0}\right)\left(x_{1}-x_{0}\right)
$$

we get

$$
\left\|F\left(x_{1}\right)\right\| \leq \frac{1}{2 b_{0}}\left\|x_{1}-x_{0}\right\|
$$

Claim. Suppose that $x_{i}, i=1,2, \ldots, n$ have been chosen such that

$$
\begin{gathered}
\left\|x_{i}-x_{0}\right\|<r_{3} \\
\left\|x_{i}-x_{i-1}\right\| \leq b_{0}\left\|F\left(x_{i-1}\right)\right\|,
\end{gathered}
$$

and

$$
\left\|F\left(x_{i}\right)\right\| \leq \frac{1}{2 b_{0}}\left\|x_{i}-x_{i-1}\right\| .
$$

Then, (8), (9) and (10) hold for $i=1,2,3, \ldots, n, \ldots$.

By (4), we have

$$
\left\|x_{n+1}-x_{n}\right\| \leq b_{0}\left\|F\left(x_{n}\right)\right\|<\frac{1}{2}\left\|x_{n}-x_{n-1}\right\|
$$

that proves (9). Also, by (11)

$$
\begin{aligned}
\left\|x_{n+1}-x_{0}\right\| & \leq\left\{\sum_{i=0}^{n} 2^{-i}\right\}\left\|x_{1}-x_{0}\right\| \\
& <\left\{1-2^{-(n+1)}\right\} r_{3}<r_{3}
\end{aligned}
$$

which proves (8).

Moreover, using (5) and

$$
F\left(x_{n+1}\right)=F\left(x_{n+1}\right)-F\left(x_{n}\right)-F^{\prime}\left(y_{n}\right)\left(x_{n+1}-x_{n}\right),
$$

we obtain

$$
\left\|F\left(x_{n+1}\right)\right\| \leq \frac{1}{2 b_{0}}\left\|x_{n+1}-x_{n}\right\|,
$$


which proves (10). The claim is now proved.

Let $n, q$ be two integers, then

$$
\begin{aligned}
\left\|x_{n+p}-x_{n}\right\| & \leq \sum_{j=1}^{q}\left\|x_{n+j}-x_{n+j-1}\right\| \\
& \leq b_{0}\left\|F\left(x_{0}\right)\right\| \cdot 2^{-n}\left\{\sum_{j=0}^{q-1} 2^{-j}\right\}<2^{-n} r_{3} .
\end{aligned}
$$

Therefore, $\left\{x_{n}\right\}, n=0,1,2, \ldots$ constitutes a Cauchy sequence in a Banach space and as such it converges to some $x^{*} \in X$. By (8) and (10) respectively, we get

$$
\left\|x^{*}-x_{0}\right\| \leq r_{3}
$$

and

$$
F\left(x^{*}\right)=0
$$

Finally, to show that $x^{*}$ is the unique solution of (2) in $U\left(x_{0}, r_{3}\right)$ let us assume that $x_{1}^{*}$ is another solution of (2) in $U\left(x_{0}, r_{3}\right)$, with $x^{*} \neq x_{1}^{*}$. Then

$$
\begin{aligned}
\left\|x^{*}-x_{1}^{*}\right\|=\left\|F^{\prime}\left(x_{0}\right)^{-1} F^{\prime}\left(x_{0}\right)\left(x^{*}-x_{1}^{*}\right)\right\| & \leq b_{0}\left\|F^{\prime}\left(x_{0}\right)\left(x^{*}-x_{1}^{*}\right)\right\| \\
& \leq \frac{1}{2}\left\|x^{*}-x_{1}^{*}\right\|,
\end{aligned}
$$

which contradicts the assumption $x^{*} \neq x_{1}^{*}$.

Therefore, $x^{*}=x_{1}^{*}$. Letting $q \rightarrow \infty$ in (12), we obtain (7) and that completes the proof of the theorem.

If $y_{n}=x_{0}, n=0,1,2, \ldots$, (1) reduces to the modified Newton's iteration which requires the evaluation of the same inverse $F^{\prime}\left(x_{0}\right)^{-1}$ at each step of the iteration.

However, if $y_{n} \neq x_{0}$, (1) requires the evaluation of the inverse operators $F^{\prime}\left(y_{n}\right)^{-1}, n=0,1,2, \ldots$ at each step, which constitutes a difficult task in general.

A usual alternative is then to find a sequence of bounded linear operators $L_{n}, n=0,1,2, \ldots$, such that

$$
\left\|L_{n}-F^{\prime}\left(x_{0}\right)\right\|<\frac{1}{4 b_{0}}
$$


and

$$
\left\|L_{n}^{-1}\right\|<b_{0}
$$

Following the proof of the above theorem, we can then easily show that the iteration

$$
x_{n+1}=x_{n}-L_{n}^{-1} F\left(x_{n}\right), \quad n=0,1,2, \ldots
$$

converges to a solution $x^{*}$ of (2).

One can refer to [5], [6] and the references there for an extensive analysis of iterations like (13).

Some of the results in [1] (especially Theorems 1 and 2) are similar to ours for $p=1$ only. However, the results there cannot be applied here for $p \neq 1$.

The motivation for the introduction of an interation like (1) when $F^{\prime}(x)$ is Hölder continuous on some open ball is due to the existence of problems like the one illustrated in the example.

Example. Consider the differential equation

$$
\begin{gathered}
x^{\prime \prime}+x^{1+p}=0, \quad p \in[0,1] \\
x(0)=x(1)=0 .
\end{gathered}
$$

We divide the interval $[0,1]$ into $n$ subintervals and we set $h=\frac{1}{n}$. Let $\left\{v_{k}\right\}$ be the points of subdivision with

$$
0=v_{0}<v_{1}<\ldots<v_{n}=1 \text {. }
$$

A standard approximation for the second derivative is given by

$$
x_{i}^{\prime \prime}=\frac{x_{i-1}-2 x_{i}+x_{i+1}}{h^{2}}, x_{i}=x\left(v_{i}\right), \quad i=1,2, \ldots, n-1 .
$$

Take $x_{0}=x_{n}=0$ and define the operator $F: \mathbb{R}^{n-1} \rightarrow \mathbb{R}^{n-1}$ by

$$
F(x)=H(x)+h^{2} \varphi(x)
$$




$$
\begin{gathered}
H=\left[\begin{array}{cccc}
2 & -1 & & \\
-1 & 2 \cdot & \cdot & 0 \\
0 & \ddots & \ddots & -1 \\
& & -1 & 2
\end{array}\right] \\
\varphi(x)=\left[\begin{array}{c}
x_{1}^{1+p} \\
x_{2}^{1+p} \\
\cdot \\
\cdot \\
\cdot \\
x_{n-1}^{1+p}
\end{array}\right]
\end{gathered}
$$

and

$$
x=\left[\begin{array}{c}
x_{1} \\
x_{2} \\
\cdot \\
\cdot \\
\cdot \\
x_{n-1}
\end{array}\right]
$$

Then

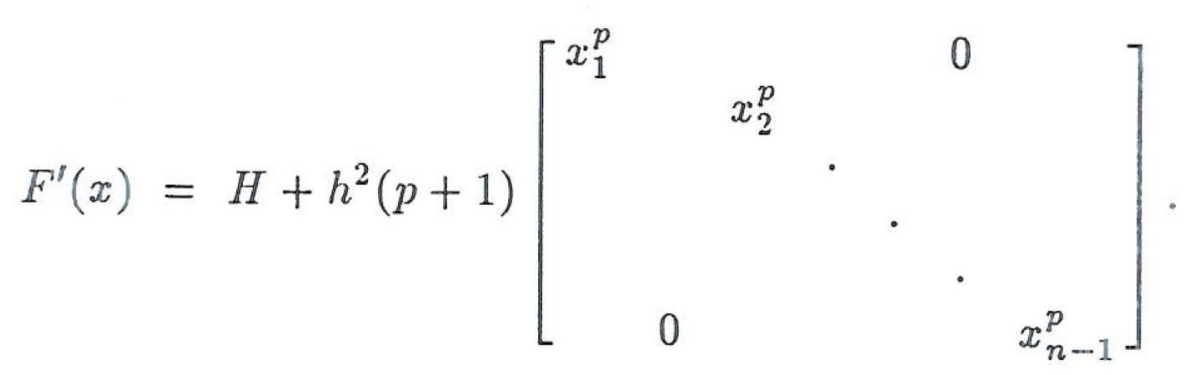

Newton's method cannot be applied to the equation

$$
F(x)=0
$$

We may not be able to evaluate the second Fréchet-derivative since it would involve the evaluation of quantities of the form $x_{i}^{-p}$ and they may not exist.

Let $x \in \mathbb{R}^{n-1}, H \in \mathbb{R}^{n-1} \times \mathbb{R}^{n-1}$ and define the norms of $x$ and $H$ by

$$
\begin{gathered}
\|x\|=\max _{1 \leq j \leq n-1}\left|x_{j}\right| \\
\|H\|=\max _{1 \leq j \leq n-1} \sum_{k=1}^{n-1}\left|h_{j k}\right| .
\end{gathered}
$$


Fог all $x, z \in \mathbb{R}^{n-1}$ for which $\left|x_{i}\right|>0,\left|z_{i}\right|>0, i=1,2, \ldots, n-1$ we obtain, for $p=\frac{1}{2}$ say,

$$
\begin{aligned}
\left\|F^{\prime}(x)-F^{\prime}(z)\right\| & =\left\|\operatorname{diag}\left\{\left(1+\frac{1}{2}\right) h^{2}\left(x_{j}^{1 / 2}-z_{j}^{1 / 2}\right)\right\}\right\| \\
& =\frac{3}{2} h^{2} \max _{1 \leq j \leq n-1}\left|x_{j}^{1 / 2}-z_{j}^{1 / 2}\right| \leq \frac{3}{2} h^{2}\left[\max \left|x_{j}-z_{j}\right|\right]^{1 / 2} \\
& =\frac{3}{2} h^{2}\|x-z\|^{1 / 2} .
\end{aligned}
$$

Therefore, under the assumptions of the theorem, iteration (1) will converge to the solution $x^{*}$ of (16).

\section{References}

[1] L. M. Graves, "Some mapping theorems", Duke Math. J., Vol 17 (1950), pp. 111-114.

[2] L. V. Kantorovich, "On Newton's method", Math. Reviews, Vol. 12 (1951), p. 419.

[3] L. V. Kantorovich and G. P. Akilov, "Functional analysis in normed spaces", Oxford Publ., Pergamon Press, 1964.

[4] I. P. Mysovkih, "On the convergence of Newton's method", Math. Reviews, Vol. 12 (1951), p.419.

[5] L. B. Rall, "Nonlinear functional analysis and applications", (Article by J. Dennis.) Academic Press, 1971.

[6] W. C. Rheinboldt, "Numerical analysis of parametrized nonlinear equations", John Wiley, Publ. 1986.

[7] L. M. Stein, "Sufficient conditions for the convergence of Newton's method in complex Banach spaces", Proc. Amer. Math. Soc. Vol. 3 (1952), pp. 858-863.

Department of Mathematics, Cameron University, Lawton, OK, 73505, U. S. A. 